\title{
Optimal Discretization Based Adaptive Finite Element Analysis for Electromagnetics with Vector Tetrahedra
}

\author{
Dennis Giannacopoulos and Steve McFee
}

\begin{abstract}
Efficient functional derivative formulas suitable for optimal discretization based refinement criteria are developed for 3-D adaptive finite element analysis (FEA) with vector tetrahedra. Results for generalized vector Helmholtz systems are derived directly from first principles, and confirmed numerically through fundamental benchmark evaluations. Practical adaption applications are illustrated for selected FEA refinement models.
\end{abstract}

Index Terms-Adaptive systems, electromagnetic analysis, error analysis, finite element methods.

\section{INTRODUCTION}

$\mathbf{T}$ HE STUDY of refinement criteria for adaptive FEA has been a subject of considerable importance and interest over the past decade [1], [2]. Today, the primary focus is on the research and development of effective and efficient techniques for practical 3-D applications [3]-[5]. Recent work has established the strengths of optimal discretization based refinement criteria, and confirmed the value of using functional gradient type error indicators for scalar electromagnetic applications [6], [7]. The theoretical potential of these variational approaches is now generally accepted [8]. The objective of this contribution is to derive, validate and evaluate a corresponding set of functional derivative formulas suitable for the development of optimal discretization based refinement criteria for 3-D adaptive FEA based on vector tetrahedra.

\section{Functional Derivatives For Vector Tetrahedra}

Adaptive FEA is especially useful for solving large problems efficiently, since the computer resources required can increase at a significant rate with respect to the problem size. For example, in some finite element implementations the approximate computational cost can be $\mathcal{O}\left(n^{3}\right)$, where $n$ is proportional to the number of degrees of freedom (DOF) used in the numerical model of the problem [9]. Today, many realistic problems require a large number of free, or unconstrained, modeling parameters in order to compute their solutions with sufficient accuracy. For instance, many practical electromagnetic systems do not possess the appropriate symmetry to allow for 1-D or 2-D treatments, and, therefore, must be analyzed using full 3-D

Manuscript received June 5, 2000.

This work was supported in part by the Natural Sciences and Engineering Research Council of Canada.

The authors are with the Department of Electrical and Computer Engineering, McGill University, Montreal, PQ, H3A 2A7, Canada.

Publisher Item Identifier S 0018-9464(01)07855-4. formulations. This has made the effective discretization of the physical problem a tacit requirement of efficient modern finite element packages. The need for such computational efficiency in finite element electromagnetics methods has led to an increased demand for advanced adaptive solver technologies.

One route to adaption which has proven to be successful for 2-D and 3-D scalar systems, is to employ local error measures closely related to the variational principle used to determine the solution to the finite element problem [6], [7]. With this type of approach, regions of inferior discretization in a finite element mesh can be detected and ranked by evaluating the sensitivity of the functional to differential displacements of the geometric nodes. Therefore, by computing the gradients of the functional with respect to vertex positions, it is possible to determine where to improve the discretization, based on a purely local error measure that is closely related to the underlying variational principle used to compute the finite element solution to the problem.

Functional gradient error indicators associated with optimal discretization based refinement criteria are defined in terms of derivatives with respect to tetrahedral vertex positions. These derivatives may be computed directly for vector Helmholtz systems. For example, in problems where the field solution variation, $\mathbf{E}$, is described in terms of the coordinate variables $x, y$, and $z$, i.e., $\mathbf{E}=\mathbf{E}(x, y, z)$, the 3 -D vector counterparts of the functional derivative formulas given in [7] for scalar systems may be derived following an analogous procedure. Consider the first-order vector tetrahedral element (linear edge element), as defined in [10], with vertex positions $\left(x_{l}, y_{l}, z_{l}\right), l=1,2,3,4$. For vector Helmholtz systems in which the true solution $\mathbf{E}$ is the stationary point of the following complex functional $F$,

$$
F(\mathbf{E})=\frac{1}{2} \int_{V}\left[\frac{1}{\mu_{r}}(\nabla \times \mathbf{E}) \cdot(\nabla \times \mathbf{E})-k_{0}^{2} \varepsilon_{r} \mathbf{E} \cdot \mathbf{E}\right] d V
$$

the $x$-, $y$-, and $z$-components, respectively, of the functional gradients may be determined from the three matrix forms:

$$
\begin{aligned}
& \frac{1}{2} E^{T} \mathbf{V} E-\frac{k_{0}^{2}}{2} E^{T} \mathbf{B} E, \\
& \frac{1}{2} E^{T} \mathbf{W} E-\frac{k_{0}^{2}}{2} E^{T} \mathbf{C} E,
\end{aligned}
$$

and

$$
\frac{1}{2} E^{T} \mathbf{P} E-\frac{k_{0}^{2}}{2} E^{T} \mathrm{D} E,
$$

evaluated over the tetrahedra that share the vertex in question. Here, $E$ is the field solution vector and $k_{0}$ is the free-space wave 
TABLE I

EDGE DEFINITIONS FOR TETRAHEDRA

\begin{tabular}{ccc}
\hline Edge $i$ & Vertex $i_{1}$ & Vertex $i_{2}$ \\
\hline & & 2 \\
2 & 1 & 3 \\
3 & 1 & 4 \\
4 & 1 & 3 \\
5 & 2 & 2 \\
6 & 4 & 4 \\
\hline
\end{tabular}

number of the system. The square matrices $\mathbf{V}, \mathbf{W}$, and $\mathbf{P}$ contain the $x$-, $y$-, and $z$-derivative information, respectively, that corresponds to the first term in (1) for vertex $l(l=1,2,3,4)$ of a tetrahedron. The entries of the matrices $\mathbf{V}, \mathbf{W}$, and $\mathbf{P}$ are defined by:

$$
\begin{aligned}
V_{i j}= & \frac{N_{i j}}{(6 \mathcal{V})^{4}}\left[\left(x_{i_{1}}-x_{i_{2}}\right) \frac{\ell_{j}}{\ell_{i}} \frac{\partial\left(x_{i_{1}}-x_{i_{2}}\right)}{\partial x_{l}} \mathcal{V}\right. \\
& \left.\quad+\left(x_{j_{1}}-x_{j_{2}}\right) \frac{\ell_{i}}{\ell_{j}} \frac{\partial\left(x_{j_{1}}-x_{j_{2}}\right)}{\partial x_{l}} \mathcal{V}-\ell_{i} \ell_{j} \frac{b_{l}}{2}\right] \\
& +\frac{\ell_{i} \ell_{j}}{(6 \mathcal{V})^{4}} \frac{\partial N_{i j}}{\partial x_{l}} \mathcal{V}, \\
W_{i j}= & \frac{N_{i j}}{(6 \mathcal{V})^{4}}\left[\left(y_{i_{1}}-y_{i_{2}}\right) \frac{\ell_{j}}{\ell_{i}} \frac{\partial\left(y_{i_{1}}-y_{i_{2}}\right)}{\partial y_{l}} \mathcal{V}\right. \\
& \left.\quad+\left(y_{j_{1}}-y_{j_{2}}\right) \frac{\ell_{i}}{\ell_{j}} \frac{\partial\left(y_{j_{1}}-y_{j_{2}}\right)}{\partial y_{l}} \mathcal{V}-\ell_{i} \ell_{j} \frac{c_{l}}{2}\right] \\
& +\frac{\ell_{i} \ell_{j}}{(6 \mathcal{V})^{4}} \frac{\partial N_{i j}}{\partial y_{l}} \mathcal{V},
\end{aligned}
$$

and

$$
\begin{aligned}
P_{i j}= & \frac{N_{i j}}{(6 \mathcal{V})^{4}}\left[\left(z_{i_{1}}-z_{i_{2}}\right) \frac{\ell_{j}}{\ell_{i}} \frac{\partial\left(z_{i_{1}}-z_{i_{2}}\right)}{\partial z_{l}} \mathcal{V}\right. \\
& \left.+\left(z_{j_{1}}-z_{j_{2}}\right) \frac{\ell_{i}}{\ell_{j}} \frac{\partial\left(z_{j_{1}}-z_{j_{2}}\right)}{\partial z_{l}} \mathcal{V}-\ell_{i} \ell_{j} \frac{d_{l}}{2}\right] \\
& +\frac{\ell_{i} \ell_{j}}{(6 \mathcal{V})^{4}} \frac{\partial N_{i j}}{\partial z_{l}} \mathcal{V}
\end{aligned}
$$

where

$\mathcal{V} \quad$ is the tetrahedral volume;

$\ell_{i} \quad$ denotes the length of the $i$ th edge connecting vertices $i_{1}$ and $i_{2}$ as given in Table I; and

$b_{i}, c_{i}$, and $d_{i}$ are geometric parameters related to the tetrahedron's vertex positions, which can be defined as follows with the subscripts progressing modulo 4 :

$$
\begin{aligned}
b_{i} & =(-1)^{i}\left|\begin{array}{lll}
1 & y_{i+1} & z_{i+1} \\
1 & y_{i+2} & z_{i+2} \\
1 & y_{i-1} & z_{i-1}
\end{array}\right|, \\
c_{i} & =(-1)^{i+1}\left|\begin{array}{lll}
1 & x_{i+1} & z_{i+1} \\
1 & x_{i+2} & z_{i+2} \\
1 & x_{i-1} & z_{i-1}
\end{array}\right|,
\end{aligned}
$$

and

$$
d_{i}=(-1)^{i}\left|\begin{array}{lll}
1 & x_{i+1} & y_{i+1} \\
1 & x_{i+2} & y_{i+2} \\
1 & x_{i-1} & y_{i-1}
\end{array}\right| \text {. }
$$

TABLE II

EXPLICIT FORMS OF $\partial b_{i} / \partial y_{l}$ IN TERMS OF $\mathcal{Z}_{i j}$

\begin{tabular}{ccccc}
$i / l$ & 1 & 2 & 3 & 4 \\
\hline & & & & \\
1 & 0 & $\mathcal{Z}_{43}$ & $\mathcal{Z}_{24}$ & $\mathcal{Z}_{32}$ \\
2 & $\mathcal{Z}_{34}$ & 0 & $\mathcal{Z}_{41}$ & $\mathcal{Z}_{13}$ \\
3 & $\mathcal{Z}_{42}$ & $\mathcal{Z}_{14}$ & 0 & $\mathcal{Z}_{21}$ \\
4 & $\mathcal{Z}_{23}$ & $\mathcal{Z}_{31}$ & $\mathcal{Z}_{12}$ & 0 \\
\hline
\end{tabular}

Further, $N_{i j}$ is defined in terms of $b_{i}, c_{i}$, and $d_{i}$ as follows:

$$
\begin{aligned}
N_{i j}= & \left(c_{i_{1}} d_{i_{2}}-d_{i_{1}} c_{i_{2}}\right)\left(c_{j_{1}} d_{j_{2}}-d_{j_{1}} c_{j_{2}}\right) \\
& +\left(d_{i_{1}} b_{i_{2}}-b_{i_{1}} d_{i_{2}}\right)\left(d_{j_{1}} b_{j_{2}}-b_{j_{1}} d_{j_{2}}\right) \\
& +\left(b_{i_{1}} c_{i_{2}}-c_{i_{1}} b_{i_{2}}\right)\left(b_{j_{1}} c_{j_{2}}-c_{j_{1}} b_{j_{2}}\right) .
\end{aligned}
$$

It may be noted that the partial derivatives of $N_{i j}$ with respect to the element vertex positions, which appear in (5)-(7), can be determined directly from (8)-(11), and Tables I and II, where the quantities $\mathcal{X}_{i j}, \mathcal{Y}_{i j}$, and $\mathcal{Z}_{i j}$ are defined as follows:

$$
\begin{aligned}
& \mathcal{X}_{i j}=x_{i}-x_{j}, \\
& \mathcal{Y}_{i j}=y_{i}-y_{j},
\end{aligned}
$$

and

$$
\mathcal{Z}_{i j}=z_{i}-z_{j} .
$$

The $x^{-}, y$-, and $z$-derivatives of the second term in the functional (1) are given by the second terms in each of (2)-(4), respectively, for vertex $l(l=1,2,3,4)$ of a tetrahedron. The entries of the matrices $\mathbf{B}, \mathbf{C}$, and $\mathbf{D}$ are defined by:

$$
\begin{aligned}
B_{i j}= & \frac{M_{i j}}{720 \mathcal{V}^{2}}\left[\left(x_{i_{1}}-x_{i_{2}}\right) \frac{\ell_{j}}{\ell_{i}} \frac{\partial\left(x_{i_{1}}-x_{i_{2}}\right)}{\partial x_{l}} \mathcal{V}\right. \\
& \left.+\left(x_{j_{1}}-x_{j_{2}}\right) \frac{\ell_{i}}{\ell_{j}} \frac{\partial\left(x_{j_{1}}-x_{j_{2}}\right)}{\partial x_{l}} \mathcal{V}-\ell_{i} \ell_{j} \frac{b_{l}}{6}\right] \\
& +\frac{\ell_{i} \ell_{j}}{720 \mathcal{V}} \frac{\partial M_{i j}}{\partial x_{l}}, \\
C_{i j}= & \frac{M_{i j}}{720 \mathcal{V}^{2}}\left[\left(y_{i_{1}}-y_{i_{2}}\right) \frac{\ell_{j}}{\ell_{i}} \frac{\partial\left(y_{i_{1}}-y_{i_{2}}\right)}{\partial y_{l}} \mathcal{V}\right. \\
& \left.+\left(y_{j_{1}}-y_{j_{2}}\right) \frac{\ell_{i}}{\ell_{j}} \frac{\partial\left(y_{j_{1}}-y_{j_{2}}\right)}{\partial y_{l}} \mathcal{V}-\ell_{i} \ell_{j} \frac{c_{l}}{6}\right] \\
& +\frac{\ell_{i} \ell_{j}}{720 \mathcal{V}} \frac{\partial M_{i j}}{\partial y_{l}},
\end{aligned}
$$

and

$$
\begin{aligned}
D_{i j}= & \frac{M_{i j}}{720 \mathcal{V}^{2}}\left[\left(z_{i_{1}}-z_{i_{2}}\right) \frac{\ell_{j}}{\ell_{i}} \frac{\partial\left(z_{i_{1}}-z_{i_{2}}\right)}{\partial z_{l}} \mathcal{V}\right. \\
& \left.+\left(z_{j_{1}}-z_{j_{2}}\right) \frac{\ell_{i}}{\ell_{j}} \frac{\partial\left(z_{j_{1}}-z_{j_{2}}\right)}{\partial z_{l}} \mathcal{V}-\ell_{i} \ell_{j} \frac{d_{l}}{6}\right] \\
& +\frac{\ell_{i} \ell_{j}}{720 \mathcal{V}} \frac{\partial M_{i j}}{\partial z_{l}},
\end{aligned}
$$

where $M_{i j}$ is given by Table III, in which $f_{i j}$ is defined in terms of $b_{i}, c_{i}$, and $d_{i}$ as follows:

$$
f_{i j}=b_{i} b_{j}+c_{i} c_{j}+d_{i} d_{j} .
$$

It may be noted that the partial derivatives of $\left(b_{m} b_{n}\right),\left(c_{m} c_{n}\right)$, and $\left(d_{m} d_{n}\right)$ with respect to the element vertex positions, which 
TABLE III EXPLICIT FORMS OF $M_{i j}$ IN TERMS OF $f_{i j}$

\begin{tabular}{cc}
\hline$i, j$ & $M_{i j}$ \\
\hline 1,1 & $2 \ell_{1}^{2}\left(f_{11}-f_{12}+f_{22}\right)$ \\
1,2 & $\ell_{1} \ell_{2}\left(f_{11}-f_{13}-f_{21}+2 f_{23}\right)$ \\
1,3 & $\ell_{1} \ell_{3}\left(f_{11}-f_{14}-f_{21}+2 f_{24}\right)$ \\
1,4 & $\ell_{1} \ell_{4}\left(f_{12}-2 f_{13}-f_{22}+f_{23}\right)$ \\
1,5 & $\ell_{1} \ell_{5}\left(2 f_{14}-f_{12}-f_{24}+f_{22}\right)$ \\
1,6 & $\ell_{1} \ell_{6}\left(f_{13}-f_{14}-f_{23}+f_{24}\right)$ \\
2,2 & $2 \ell_{2}^{2}\left(f_{11}-f_{13}+f_{33}\right)$ \\
2,3 & $\ell_{2} \ell_{3}\left(f_{11}-f_{14}-f_{13}+2 f_{34}\right)$ \\
2,4 & $\ell_{2} \ell_{4}\left(2 f_{12}-f_{13}-f_{23}+f_{33}\right)$ \\
2,5 & $\ell_{2} \ell_{5}\left(f_{14}-f_{12}-f_{34}+f_{23}\right)$ \\
2,6 & $\ell_{2} \ell_{6}\left(f_{13}-2 f_{14}-f_{33}+f_{34}\right)$ \\
3,3 & $2 \ell_{3}^{2}\left(f_{11}-f_{14}+f_{44}\right)$ \\
3,4 & $\ell_{3} \ell_{4}\left(f_{12}-f_{13}-f_{24}+f_{34}\right)$ \\
3,5 & $\ell_{3} \ell_{5}\left(f_{14}-2 f_{12}-f_{44}+f_{24}\right)$ \\
3,6 & $\ell_{3} \ell_{6}\left(2 f_{13}-f_{14}-f_{34}+f_{44}\right)$ \\
4,4 & $2 \ell_{4}^{2}\left(f_{22}-f_{23}+f_{33}\right)$ \\
4,5 & $\ell_{4} \ell_{5}\left(f_{23}-f_{22}-2 f_{34}+f_{24}\right)$ \\
4,6 & $\ell_{4} \ell_{6}\left(f_{23}-2 f_{24}-f_{33}+f_{34}\right)$ \\
5,5 & $2 \ell_{5}^{2}\left(f_{22}-f_{24}+f_{44}\right)$ \\
5,6 & $\ell_{5} \ell_{6}\left(f_{24}-2 f_{23}-f_{44}+f_{34}\right)$ \\
6,6 & $2 \ell_{6}^{2}\left(f_{33}-f_{34}+f_{44}\right)$ \\
\hline
\end{tabular}

TABLE IV

EXPLICIT FORMS OF $\partial\left(b_{m} b_{n z}\right) / \partial y_{l}$ IN TERMS OF $b_{i}$ AND $\mathcal{Z}_{i j}$

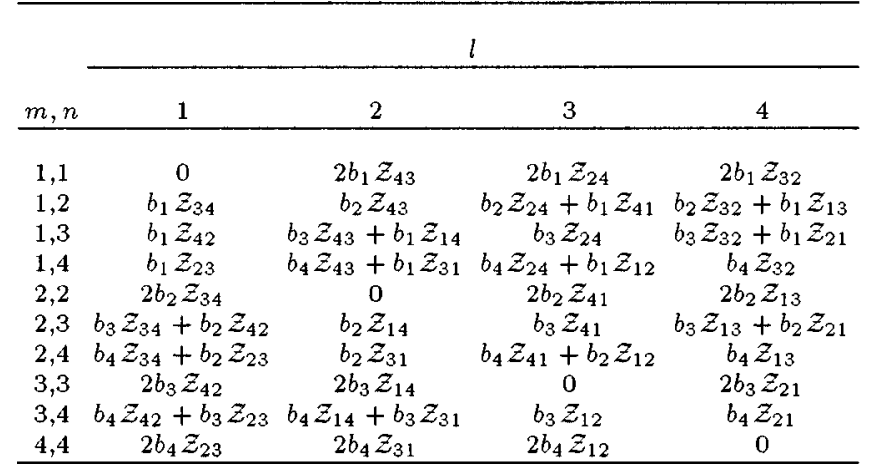

are implicit in (15)-(17), can be determined directly from (8)-(10), and are given for reference in Table IV.

Once the gradients of the functional with respect to vertex positions have been computed, they may be used in various ways as error indicators. One simple approach is to assess a weighted sum of the vertex-based functional gradients for each element, then use these values to rank the elements for refinement. In this study, this method is investigated to illustrate one possible way to exploit the new 3-D refinement criteria proposed for vector adaptive finite element solvers.

\section{RESULTS}

In order to validate the functional derivative formulas derived above, the $x$-component formulas were tested with a benchmark evaluation based on the geometry and mesh defined by Fig. 1 .

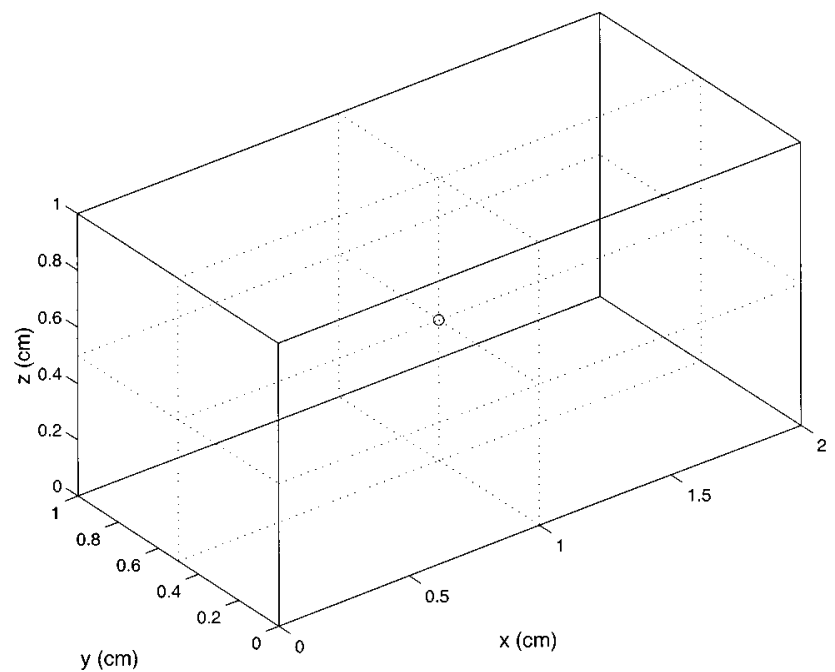

Fig. 1. Hexahedra used to define tetrahedral mesh for cavity.

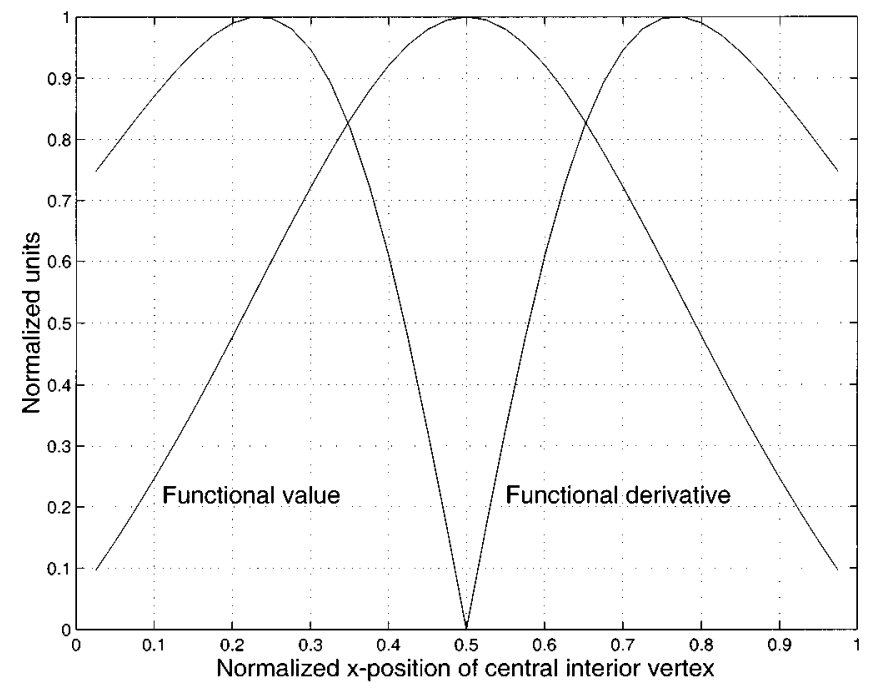

Fig. 2. Variation of normalized functional value and separately normalized magnitude of functional derivative with $x$-position of central interior vertex.

The system consists of an air-filled rectangular cavity with perfectly conducting walls and excited at the $\mathrm{TE}_{101}$ resonant frequency. One-half of the cavity was discretized using 40 tetrahedra based on subdividing each of the 8 hexahedra shown in Fig. 1 into five tetrahedra - the symmetry plane is defined by $z=0$ between the conductors. The full cavity has dimensions $2 \mathrm{~cm}, 1 \mathrm{~cm}$, and $2 \mathrm{~cm}$ in the $x, y$ and $z$ directions, respectively, and was analyzed using first-order vector tetrahedra (linear edge elements) [10]. The functional derivative correctly identified the optimal horizontal position for the central interior vertex, to yield the stationary value of the functional (Fig. 2). Furthermore, the $y$ - and $z$-components of the functional gradients were confirmed to evaluate numerically to zero at the optimal $y$ and $z$ positions for the central interior vertex, respectively, corresponding to the stationary value of the functional.

The proposed 3-D adaptive refinement criterion was also evaluated using the benchmark problem described by Fig. 1 and excited at the $\mathrm{TE}_{101}$ resonant frequency. Performance results for the first-order $h$-adaption studies (B) on functional 


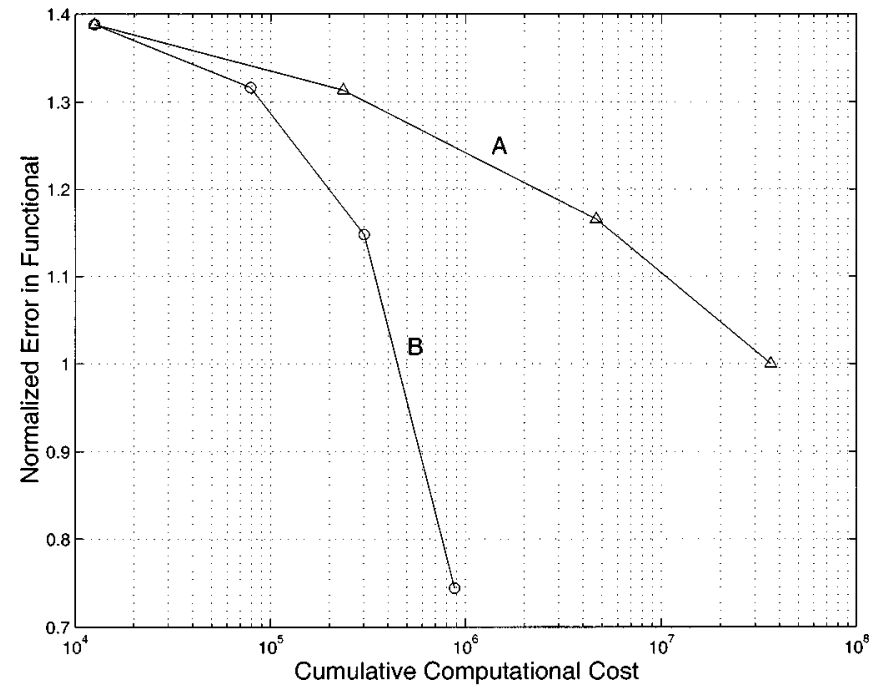

Fig. 3. Cumulative cost of adaption versus error in functional.

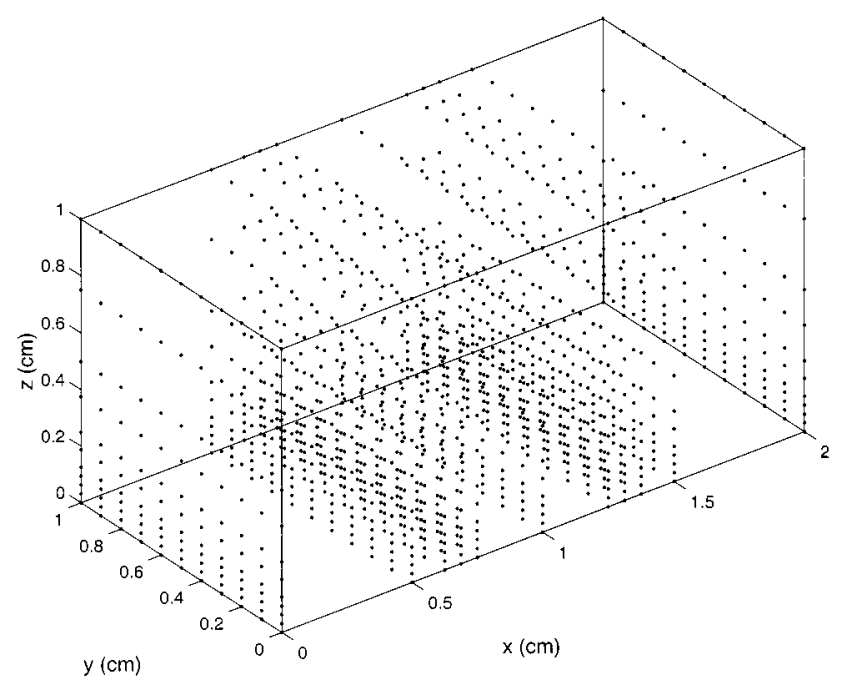

Fig. 4. Sample vertex distribution for adaptively refined mesh.

convergence are presented in Fig. 3. The uniform $h$-refinement baseline (A) is included for comparison. It may be noted that the $h$-adaption performance results indicate a considerable savings in computational cost relative to the uniform refinement approach. In order to illustrate the focus of DOF produced by the new refinement criterion, an example $h$-refined mesh is represented in Fig. 4, in terms of the distribution of tetrahedra vertices; for comparison, an example uniform refinement mesh with the same number of elements is represented by Fig. 5. A $200 \%$ increment in the number of DOF per adaptive step was used to update the $h$-adaption discretizations based on the new refinement criterion for this benchmark problem.

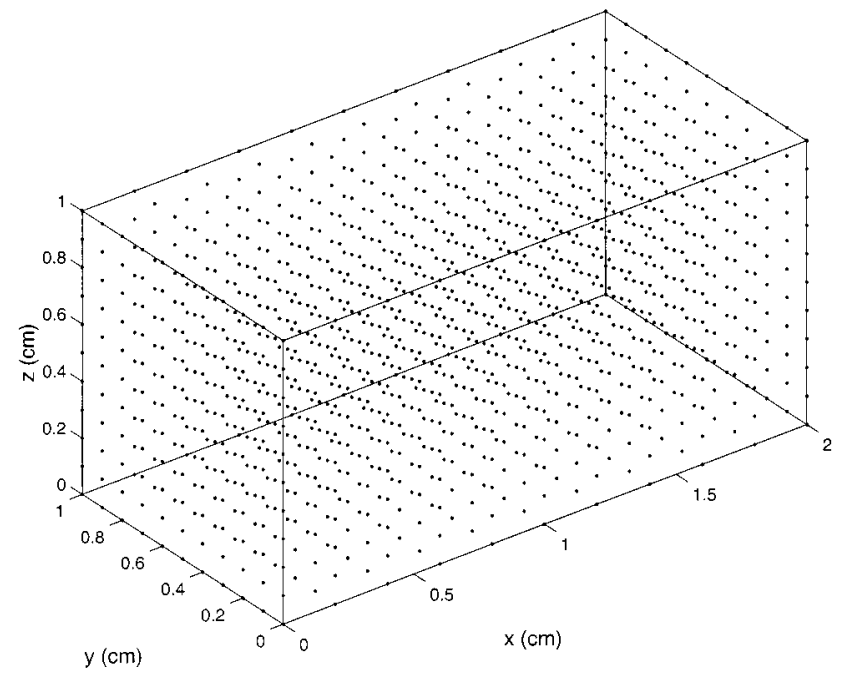

Fig. 5. Sample vertex distribution for uniformly refined mesh.

\section{CONCLUSIONS}

New functional derivative formulas suitable for optimal discretization based refinement criteria with linear edge elements for 3-D vector Helmholtz FEA have been derived. The formulas for the gradients of the functional with respect to tetrahedra vertex positions were validated by tests based on a simple 3-D benchmark system. The $h$-adaption performance results for the benchmark system that was investigated show that the new refinement criteria can be successfully used in 3-D adaptive finite element solvers to effectively and economically distribute DOF over the problem domain.

\section{REFERENCES}

[1] P. Fernandes, P. Girdinio, P. Molfino, and M. Repetto, "Local error estimates for adaptive mesh refinement," IEEE Trans. Magn., vol. 24, no. 1, pp. 299-302, 1988.

[2] D. Giannacopoulos and S. McFee, "Toward optimal $h-p$ adaption near singularities in finite element electromagnetics," IEEE Trans. Magn., vol. 30, no. 5, pp. 3523-3526, 1994.

[3] L. Janicke and A. Kost, "Error estimation and adaptive mesh generation in the 2D and 3D finite element method," IEEE Trans. Magn., vol. 32, no. 3, pp. 1334-1337, 1996.

[4] N. A. Golias and T. D. Tsiboukis, "Adaptive refinement strategies in 3D,” IEEE Trans. Magn., vol. 29, no. 2, pp. 1886-1889, 1993.

[5] J. P. Webb and B. Forghani, "Adaptive Improvement of magnetic fields using hierarchal tetrahedral finite elements," IEEE Trans. Magn., vol. 30, no. 5, pp. 3511-3514, 1994.

[6] S. McFee and D. Giannacopoulos, "Optimal discretization based refinement criteria for finite element adaption," IEEE Trans. Magn., vol. 32, no. 3, pp. 1357-1360, 1996.

[7] - "Functional derivatives and optimal discretization based refinement criteria for adaptive finite element analysis with scalar tetrahedra," IEEE Trans. Magn., vol. 35, no. 3, pp. 1326-1329, 1999.

[8] L. Y. Li and P. Bettess, "Notes on mesh optimal criteria in adaptive finite element computations," Com. Num. Meth. Eng., vol. 11, pp. 911-915, 1995.

[9] P. P. Silvester and R. L. Ferrari, Finite Elements for Electrical Engineers, 2nd ed. Cambridge: Cambridge University Press, 1990.

[10] J. Jin, The Finite Element Method in Electromagnetics. New York: John Wiley and Sons, Inc., 1993. 